HNO 2009 • 57:974-974

DOI 10.1007/s00106-009-1984-2

Online publiziert: 23. August 2009

(c) Springer Medizin Verlag 2009

P.A. Federspil

Univ.-Hals-Nasen-Ohren-Klinik, Universitätsklinikum Heidelberg

\title{
Neue Entwicklungen in der computerassistierten Chirurgie
}

CAS in der HNO-Heilkunde, Kopf- und Halschirurgie geben, verbunden mit einem Ausblick auf zukünftige Entwicklungen wie bspw. der Robotik. Mit Prof. Marco Caversaccio, Klinikdirektor der Universitäts-HNO-Klinik am Inselspital in Bern, und Privatdozent Dr. Gero Strauss, Ltd. Oberarzt an der Universitätsklinik Leipzig, konnten ausgewiesene Koryphäen auf dem Gebiet der CAS und Robotik gewonnen werden. Die Anwendungen der CAS an der lateralen Schädelbasis werden von Prof. Caversaccio ausgeführt. Privatdozent Dr. Strauss gibt ein Update über den Bereich der vorderen Schädelbasis. Abschließend wird noch über neue Entwicklungen der computerassistierten Chirurgie von der intraoperativen Bildgebung bis zur ultraschallbasierten Navigation berichtet. Ich glaube, dass Sie als Leser der HNO damit einen abgerundeten Überblick zum Thema CAS in der HNO-Heilkunde gewinnen werden.

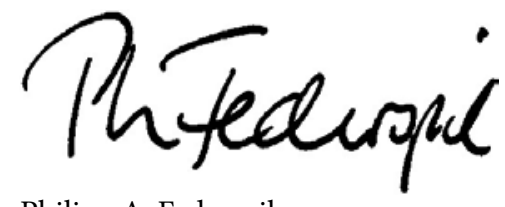

Philipp A. Federspil

pitertechnologie mit immer schnellerer Prozessorleistungen. Aber auch Fortschritte im Bereich der angrenzenden $\mathrm{Ge}$ biete wie der diagnostischen und intraoperativen Bildgebung sowie der Bildanzeige verbessern die CAS. „High definition" (HD) ist im Consumerbereich angekommen und damit auch für die Medizintechnologie erschwinglich geworden.

Diese Ausgabe der HNO soll einen Überblick über den aktuellen Stand der

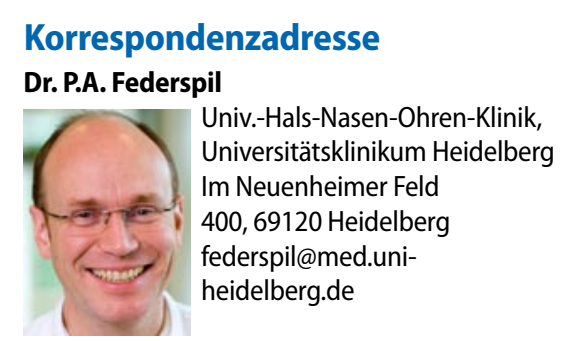

Literatur

1. Caversaccio M, Bachler R, Ladrach K et al (2000) Frameless computer-aided surgery system for revision endoscopic sinus surgery. Otolaryngol Head Neck Surg 122:808-813

2. Caversaccio M, Zheng G, Nolte LP (2008) Computerassistierte Chirurgie der Nasennebenhöhlen und der vorderen Schädelbasis. HNO 56:376-378, 372-780

3. Eggers G, Kress B, Muhling J (2008) Automated registration of intraoperative $C T$ image data for navigated skull base surgery. Minim Invasive Neurosurg 51:15-20

4. Eggers G, Muhling J, Marmulla R (2006) Image-topatient registration techniques in head surgery. Int J Oral Maxillofac Surg 35:1081-1095

5. Heermann R, Schwab B, Issing PR et al (2001) Image-guided surgery of the anterior skull base. Acta Otolaryngol 121:973-978

6. Schlöndorff G, Mösges R, Meyer-Ebrecht D et al (1989) CAS (computer assisted surgery). Ein neuartiges Verfahren in der Kopf- und Halschirurgie. HNO 37:187-190

7. Schramm A, Gellrich NC, Gutwald R et al (2000) Indications for computer-assisted treatment of cranio-maxillofacial tumors. Comput Aided Surg 5:343-352 